\title{
Using large surveys, multiwavelength catalogs, and databases for new discoveries
}

\author{
A.M. Mickaelian ${ }^{a}$, L.A. Sargsyan ${ }^{a}$, G.A. Mikayelyan ${ }^{b}$ \\ ${ }^{a}$ Byurakan Astrophysical Observatory (BAO), Armenia \\ ${ }^{b}$ Yerevan State University (YSU), Armenia \\ E-mail: aregmick@aras.am, sarl11@yahoo.com, gormick@mail.ru
}

During the recent years, large amounts of astronomical data have been obtained by ground-based and space telescopes covering all wavelength ranges, some of them being all-sky or large-area surveys covering dozens of thousands square degrees (ROSAT, XMM, and Chandra in X-ray; GALEX in UV; SDSS, USNO, MAPS, APM, and GSC in optical; 2MASS in NIR; IRAS, ISO, SST, and AKARI in IR/FIR; and NVSS and FIRST in radio). In addition, large-area spectroscopic surveys, such as FBS/SBS and HQS/HES have been digitized and are available as well. At present all they are stored in various databases, most of them giving VO access to the data. This makes possible not only the open access but also a fast analysis and managing of these data. By an analysis and matching data from various surveys and catalogs, one can find out a data set that itself is new, though published long ago in different catalogs. New discoveries this way become possible.

A good example was our analysis of the IRAS-FSC catalog for extragalactic sources that have very high IR/opt flux ratio. Among the 173,044 FSC sources, 2310 were found having quality flags 2 or 3 for both 60 and $100 \mu$ fluxes and a counterpart among the 811,117 FIRST radio catalog sources. This cross-correlation resulted in revealing the extragalactic sources and more accurate FIRST coordinates to find the genuine optical counterparts. Later on, optical identification of these sources revealed faint counterparts (for optical magnitudes, we used MAPS and SDSS data) and those with record IR/opt flux ratios (between 43 and 1110) were found. Spitzer IRS observations discovered 28 new ULIRGs and AGN among them (Sargsyan et al., 2008, ApJ 683, 114). Thus, dozens of thousands sources hided a few very interesting ones that needed to be discovered.

It is shown how the discoveries come when a comparative analysis of data from various catalogs and databases and their cross-correlation is made. It is important to find out, which particular data should be matched to reveal new properties of objects or obtain new relations between them. However, one must be careful with inhomogeneity and selection effects and have to make necessary correction and transformations. In addition, new efficient methods and software for analysis of large catalogs and building multiwavelength (MW) SEDs for objects are needed such as VO tools. A revision of classifications based on MW SEDs in the nearest future will provide a new vision on various objects and phenomena.

Accelerating the Rate of Astronomical Discovery, sps5

August 11-14, 2009

Rio de Janeiro, Brazil 